\title{
Congenital toxoplasmosis transmitted by human immunodeficiency-virus infected women
}

Authors

Kátia Martins Lopes de

Azevedo

Sérgio Setúbal

Vania Gloria Silami

Lopes $^{3}$

Luiz Antônio Bastos

Camacho $^{4}$

Solange Artimos de

Oliveira $^{5}$

${ }^{1} \mathrm{MD}$ - Discipline of Infectious and Parasitic Diseases,

Hospital Universitário Antônio Pedro, Universidade

Federal Fluminense, Niterói,

RJ, Brazil.

${ }^{2} \mathrm{MD}, \mathrm{PhD}$ - Discipline of

Infectious and Parasitic

Diseases, Hospital

Universitário Antônio

Pedro, Universidade Federal

Fluminense, Niterói, RJ, Brazil.

${ }^{3} \mathrm{MD}, \mathrm{PhD}$ - Department

of Pathology, Hospital

Universitário Antônio

Pedro, Universidade Federal

Fluminense, Niterói, RJ, Brazil.

${ }^{4} \mathrm{MD}, \mathrm{PhD}$ - Escola Nacional

de Saúde Pública, Fundação

Oswaldo Cruz, Rio de Janeiro,

Brazil

${ }^{5} \mathrm{MD}, \mathrm{PhD}$ - Discipline of

Infectious and Parasitic

Diseases, Hospital

Universitário Antônio

Pedro, Universidade Federal

Fluminense, Niterói, RJ, Brazil.

Submitted on: $02 / 02 / 2009$

Approved on: 05/27/2009

Correspondence to:

Kátia Martins Lopes de

Azevedo, MD

Disciplina de Doenças

Infecciosas e Parasitárias,

Hospital Universitário

Antônio Pedro

Rua Marquês do Paraná,

$303,2^{\circ}$ andar

Niterói - RJ - Brazil

CEP: 24030210

Phone/Fax: 5521

26299311

E-mail:kmlazevedo@

yahoo.com.br

This study received

financial support from

the Conselho Nacional de

Desenvolvimento Científico

e Tecnológico $(\mathrm{CNPq})$

(references 302430/2002-4

and $471155 / 03-9)$

\begin{abstract}
We report the occurrence of congenital toxoplasmosis in three infants born to HIV infected women who had high anti-toxoplasma IgG and negative IgM during pregnancy. We briefly reviewed available literature and discussed the possible transmission mechanisms of congenital toxoplasmosis among HIV infected pregnant women. Serum samples were tested for Toxoplasma gondii IgM and IgG antibodies using commercial enzyme immunoassay and IgG-avidity tests. In the first case, fetal death occurred at 28 th week of gestation. In the second case, congenital toxoplasmosis was diagnosis at 6 th month of life; and in the third case, an HIV-infected newborn, congenital toxoplasmosis was asymptomatic. These cases point out to the possibility of enhanced maternal-fetal transmission of T. gondii infection by HIV-infected women chronically infected, which may have important public health consequences, considering that increasing frequency of HIV-infection has been observed among women of childbearing age around the world.
\end{abstract}

Keywords: congenital toxoplasmosis, human immunodeficiency-virus, prevention, AIDS.

[Braz J Infect Dis 2010;14(2):186-189] @Elsevier Editora Ltda.

\section{INTRODUTION}

Toxoplasma gondii is a ubiquitous obligatory widely-distributed intracellular protozoan. Once infected, the host normally acquires lifelong immunity induced by the persistence of the parasite in an encysted form. Maternal immunity appears to protect against fetal infections. If anti- $T$. gondii IgG antibody is confirmed before pregnancy, then the woman is not at risk for a congenitally infected fetus. Congenital toxoplasmosis is usually the result of maternal acquisition of $T$. gondii for the first time during gestation. ${ }^{1}$ However, vertical transmission of toxoplasmosis as a result of reactivation has been described in immunocompromised women ${ }^{2-4}$ and more rarely in immunocompetent women. ${ }^{5}$

Clinical manifestations of congenital toxoplasmosis are numerous, including intracranial calcifications, convulsion, psychomotor retardation, strabismus, chorioretinitis, microcephaly, and hydrocephaly observed in infancy or later. However, most neonates with congenital toxoplasmosis are asymptomatic or with subclinical presentation of the disease at birth. ${ }^{6}$

There is limited data on medical literature about the frequency of $T$. gondii vertical transmission among HIV-infected women. ${ }^{7-10}$ In
Brazil, the prevalence of seropositivity for $T$. gondii is $50 \%$ to $80 \%$ in women of childbearing age. ${ }^{11-15}$ Although T. gondii infection is highly prevalent in Brazil, there are only two reports of congenital toxoplasmosis documented among HIV-infected women. ${ }^{8,16}$

In this paper we describe serological and clinical findings of three cases of congenital toxoplasmosis diagnosed among 113 HIV-exposed infants followed at the Infectious Diseases Department of Antônio Pedro University Hospital, since 1998, in Niterói, Rio de Janeiro, Brazil. Seventy per cent of HIV-infected mothers had serologic evidence of anti-T. gondii IgG during pregnancy. Blood samples were taken serially for HIV routine diagnostic purposes, and some serum samples from the three patients were tested retrospectively for the present study.

\section{CASES REPORT}

First case: A 26-year-old caucasian woman was diagnosed with HIV-infection in May 2004, at 14 weeks' gestation of her third pregnancy. Routine prenatal testing revealed Anti-T. gondii IgG positive and anti-T. gondii IgM negative. She was asymptomatic and had never 
been treated with antiretroviral drugs. Initial CD4 cell count was $291 / \mathrm{mm}^{3}$ and viral load was 65,000 copies/mL (4.81 $\log$ ) by nucleic acid sequence based amplification (NASBA). From the $22^{\text {nd }}$ week of gestation onwards, she was started on zidovudine, lamivudine and nevirapine for prophylaxis of HIV-infection vertical transmission. At 21 weeks' gestation (July 2004) another toxoplasma serology was done and the result revealed IgG positive $(2,320 \mathrm{IU} / \mathrm{mL}$; cut-off: $8 \mathrm{IU} /$ $\mathrm{mL}$ ) and IgM negative by enzyme linked fluorescent assay (ELFA) (VIDAS ${ }^{\circledR}$ Toxo IgG II and IgM, Biomérieux SA, France). The anti-T. gondii IgG-avidity was high (0.586; cutoff: 0.3) (VIDAS ${ }^{\circledR}$ Toxo IgG Avidity, Biomérieux SA). At 28 weeks' gestation (August 2004), she was admitted to the hospital with fever, flank pain, and pyuria. She received cefazoline for treatment of urinary tract infection. Her laboratory exams showed anaemia, and toxoplasma serology was positive for IgG $(2,488 \mathrm{IU} / \mathrm{mL})$ and negative for IgM antibodies by ELFA. Ultrasonography revealed fetal death and delivery was induced. Fetal autopsy showed hepatosplenomegaly and anasarca. Microscopic analysis of heart sections stained with hematoxylin and eosin (HE) revealed pericarditis and myocarditis, with acute and chronic inflammatory infiltration and micro-elements morphologically compatible with T. gondii trophozoites. Placental histology showed diffuse necrotizantis villositis with trophozoites. Immunostaining with polyclonal antibody anti-T. gondii (rabbit anti-T. gondii; code no.: B1013, Dako, Denmark) of heart and placenta tissue sections confirmed HE microscopic analysis. Her toxoplasma serology repeated in October 2004 was positive for IgG antibodies $(2,262 \mathrm{IU} / \mathrm{mL})$ by ELFA. Six months after, the IgG titre by ELFA was lower $(1,232 \mathrm{IU} / \mathrm{mL})$. Both sera were negative with the IgM-ELFA and showed high avidity IgG antibodies.

Second case: A 30-year-old caucasian woman was diagnosed with HIV-infection in September 2005, on the $12^{\text {th }}$ week of her third pregnancy. Routine prenatal testing revealed anti- $T$. gondii IgG positive (1/512; cut-off: $1 / 16)$ and IgM negative by EIA. Eight months before this pregnancy, she had done a toxoplasma serology with equal result. In November 2005, on the $20^{\text {th }}$ week of gestation, she was asymptomatic and her CD4 cell count was $147 / \mathrm{mm}^{3}$ and the viral load was 750 copies $/ \mathrm{mL}(2.87 \mathrm{log})$ by NASBA. Her toxoplasma serology was positive for $\operatorname{IgG}(364 \mathrm{IU} / \mathrm{mL})$ and negative for IgM by ELFA. The VIDAS IgG-avidity test was high (0.620). From the 21th week of gestation onwards, she was started on zidovudine, lamivudine, and nevirapine for treatment and also for prophylaxis of HIV-infection vertical transmission. At 32 weeks' gestation (February 2006), her CD4 count was $213 / \mathrm{mm}^{3}$, viral load was below 80 copies/ $\mathrm{mL}$ by NASBA and toxoplasma serology was positive for IgG antibodies $(476 \mathrm{IU} / \mathrm{mL}$ ) and negative for IgM by ELFA. The VIDAS IgG-avidity test was high (0.519). At 39 weeks' gestation (March 2006), she was submitted to a cesarean-sec- tion because of fetal suffering. An apparently healthy girl was born, and zidovudine was given to the newborn for 6 weeks to prevent HIV infection. The baby was followed up by the Pediatric Department and in June 2006 she started presenting nistagmus. On clinical exam there were strabismus, neurodevelopmental delay and hepatosplenomegaly. Laboratory screening revealed anti-T. gondii IgG $(107 \mathrm{IU} / \mathrm{mL})$ and a positive IgM by ELFA. Funduscopy showed chorioretinitis in her left eye and the skull radiography was normal. The child was treated with sulfadiazine, pirimetamine, and folinic acid for 6 months. During follow-up visits, chorioretinal scars were seen in funduscopic reevaluation and intracranial calcifications on brain CT scan. Anti-T. gondii serology by ELFA revealed IgG positive (99 IU/mL) and IgM negative. The child had a serology for HIV negative at the $11^{\text {th }}$ month of life, being considered HIV-uninfected. Placenta histology showed intervillositis, extensive villitis and chorioamnionitis.

Retrospectively, the mother's serology showed a significant increase in Anti-T. gondii IgG titre by ELFA from 364 $\mathrm{IU} / \mathrm{mL}$ in November 2005 to 3,616 IU/mL in March 2006. Both sera were negative with the IgM-ELFA and had high avidity IgG antibodies. During follow-up visits, maternal anti-T. gondii serology still showed high levels of IgG antibodies detected by ELFA: July 2006 - IgG: 6,138 IU/mL; January 2007 - IgG: 2,970 IU/mL; October 2007 - IgG: $2,188 \mathrm{IU} / \mathrm{mL}$. All serum samples tested for IgM antibodies by ELFA were negative and also had high avidity IgG antibodies anti-T. gondii.

Third case: A 16-year-old afro-descend adolescent was diagnosed with HIV-infection in 2003 during her first pregnancy. Her follow-up visits were at irregular intervals, and antiretrovirals were given only for prophylaxis of HIV-infection vertical transmission during pregnancy. Her toxoplasma serology had always been positive for IgG and negative for IgM antibodies since 2003. In February 2008, she sought for medical advice as she was at 33 weeks' gestation. She had pruritus, anaemia, and her toxoplasma serology was positive for IgG antibodies (204 IU/mL) and inconclusive for IgM by ELFA. In March 2008 (36 weeks' gestation), she complained of progressive visual impairment affecting the left eye, and ophthalmologic examination showed uveitis due to toxoplasmosis. Her toxoplasma serology was positive for IgG antibodies (198 IU/mL) and negative for IgM by ELFA. The VIDAS IgG-avidity test was high (0.391). She was treated with sulfadiazine, pirimetamine, and folinic acid for ocular toxoplasmosis and zidovudine for prophylaxis of HIV-infection vertical transmission. Her CD4 cell count was $171 / \mathrm{mm}^{3}$ and the viral load was 333,129 copies $/ \mathrm{mL}(5.523 \mathrm{log})$ by b-DNA technique. She was submitted to an elective cesarean-section at 38 weeks' gestation. A healthy girl was born, and laboratory showed toxoplasma serology positive for IgG antibodies (172 IU/mL) and positive for IgM by ELFA. Cerebrospinal 
fluid examination reveled pleocytosis ( $20 \mathrm{cell} / \mathrm{mm}^{3}, 100 \%$ neutrophil predominance), elevated protein $(129 \mathrm{mg} / \mathrm{dL})$. Funduscopy and skull radiography were normal. The child was discharged from hospital receiving drugs for treatment of congenital toxoplasmosis and to prevent HIV infection. The baby was followed up by the Pediatric Department. In May 2008, her HIV viral load was positive (163,000 copies $/ \mathrm{mL} ; 5.21 \mathrm{log}$ ) by b-DNA technique. In June 2008, she had oral candidiasis and her CD4 cell count and viral load were, respectively, $1,407 / \mathrm{mm}^{3}$ and more than 500,000 copies $/ \mathrm{mL}$. Her clinical and laboratorial status was compatible with aids, and antiretroviral treatment was started in July 2008.

\section{DISCUSSION}

Although not proved, our first case was possibly a reactivation of latent $T$. gondii infection. There was a possibility of acquired infection in the first 14 weeks of gestation; since absence of specific IgM is characteristic for HIV-infected patients. ${ }^{8}$ Fetal death may support this hypothesis of newly acquired infection. However, the sharp rise in specific IgG suggests remote infection, because in HIV-infected patients the serum levels of antibodies against existing infections increase, but the humoral response to recently introduced antigens decreases. ${ }^{17}$

Our first case indicates that congenital toxoplasmosis may occur in children of HIV-positive mothers who are not severely immunocompromised as a result of toxoplasmic reactivation. Even though no serology to T. gondii infection was available before the present gestation, negative IgM and high IgG-avidity antibodies are compatible with primary infection before the pregnancy, although HIV-infected pregnant women may present newly acquired $T$. gondii infection without seroconvertion to specific IgM. ${ }^{9}$ The other two cases were both severely immunocompromised and their antibody test results clearly showed $T$. gondii infection acquired before pregnancy. In the three cases, congenital toxoplasmosis was probably due to reactivation of latent maternal infection, although reinfection with a different strain of $T$. gondii is also possible. $^{2}$

The second case alerts for the necessity of doing tests for congenital infections in newborns of HIV-infected mothers. The opportunity of treating infant since birth, or even before birth, must not be lost. This case, in which CT scan showed intracerebral calcifications, alerts to very low sensitivity of skull radiography to congenital toxoplasmosis. ${ }^{18}$ This infant was treated for only 6 months because when she was 1-yearold there were no signs of active infection.

Although HIV-infected pregnant women chronically infected with T. gondii may transmit the infection to the fetus, the risk is low and certainly less than $5 \%{ }^{7,8,10}$ Some authors advocate that women with CD4 less than 100 cells/ $\mathrm{mm}^{3}$ who are Anti-T. gondii IgG positive should be treated with adequate prophylaxis during pregnancy to attempt to interrupt transmission of $T$. gondii from chronically infected women to fetus. They suggest this advice may be also appropriate for HIV-uninfected patients who are severely immunocompromised for other reason. ${ }^{10}$ Other study suggests that the risk of maternal-fetal transmission is not sufficient high to justify routine anti-toxoplasmosis chemoprophylaxis for HIV-infected pregnant women, although the authors highlight the need of further research on this issue. $^{7}$

The lack of an established risk of maternal-fetal infection, particularly in countries like Brazil where there are high seroprevalence rates of $T$. gondii, may explain the current obstetric management of these patients without the prescription of anti-toxoplasmosis chemoprophylaxis in chronically infected women.

Our report shows the need for special attention to maternal titles of anti-T. gondii antibodies during HIV prenatal care even in women chronically infected with T. gondii and also in those not severely immunocompromised. A high concentration of T. gondii-specific IgG and a positive result for T. gondii-specific IgM in immunocompetent patients usually indicate recent infection, because T. gondiispecific IgG increases rapidly in the acute infection. Conversely, a low concentration of $T$. gondii-specific IgG and no T. gondii-specific IgM is a sign a remote infection. ${ }^{19}$ These indications may not apply to HIV-infect pregnant women. If, as described for HIV-infected patients in general, ${ }^{17}$ it is true for HIV-infected pregnant women, the serum levels of antibodies against existing infections increase and the humoral response to recently introduced antigens decreases, then, high T. gondii-specific IgG concentrations may, paradoxically, indicate latent T. gondii infection, whereas low concentrations may indicate recent infection, when the risk of congenital toxoplasmosis may be high. A higher risk of congenital toxoplasmosis with low T gondii-specific IgG concentration and negative specific IgM is worth of attention in order to not let a case of acute gestational toxoplasmosis unidentified in HIV-infected pregnant women. It also enhances the relevance of routine antibody screening for congenital toxoplasmosis in the newborn blood. The screening for IgM antibody is an important tool for the detection of newborns infected with T. gondii ${ }^{20}$ and it is mandatory in HIV-exposed infants. ${ }^{2}$

Considering the increasing frequency that HIV-infection has been observed among women of childbearing age around the world and the severity of fetal lesions caused by congenital toxoplasmosis, the possibility of enhanced maternal-fetal transmission of this infection by HIV-infected women chronically infected with $T$. gondii may have important public health consequences, mainly in those countries with high seroprevalence rates of T. gondii infection. 


\section{ACKNOWLEDGEMENTS}

We would like to thank Dr. Rosa Vieira and her team, from the Immunology Laboratory, Hospital Universitário Antônio Pedro (UFF), for carrying out the serological tests for toxoplasmosis.

\section{REFERENCES}

1. American College of Obstetricians and Gynecologists. Perinatal viral and parasitic infections. ACOG Pract Bull 2000; 20.

2. Bachmeyer C, Mouchnino G, Thulliez P, Blum L. Congenital toxoplasmosis from an HIV-infected woman as a result of reactivation. J Infect 2006; 52:e55-e57.

3. Mitchell CD, Erlich SS, Mastrucci MT et al. Congenital toxoplasmosis occurring in infants perinatally infected with human immunodeficiency virus 1. Pediatr Infect Dis J 1990; 9:512-8.

4. Marty P, Bongain A, Loiseau S et al. Lethal congenital toxoplasmosis resulting from reactivation of toxoplasmosis in a pregnant HIV-positive patient. Presse Med 2002; 31:1558.

5. Kodjikian L, Hoigne I, Adam $\mathrm{O}$ et al. Vertical transmission of toxoplasmosis from a chronically infected immunocompetent woman. Pediatr Infect Dis J 2004; 23(3):272-4.

6. Sáfadi MA, Berezin EN, Farhat CK, Carvalho ES. Clinical presentation and follow up of children with congenital toxoplasmosis in Brazil. Braz J Infect Dis 2003; 7(5):325-31.

7. European Colaborative Study and Research Network on Congenital Toxoplasmosis. Low incidence of congenital toxoplasmosis in children born to women infected with human immunodeficiency virus. Eur J Obstet Gynecol Reprod Bio 1996; 68:93-6.

8. Lago EG, Conrado GS, Piccoli CS, Carvalho RL, Bender AL. Toxoplasma gondii antibody profile in HIV-infected pregnant women and the risk of congenital toxoplasmosis. Eur J Clin Microbiol Infect Dis 2008; [epud ahead of print].

9. Nogueira SA, Machado ES, Costa TP et al. Co-infection with Toxoplasma gondii in a cohort of HIV infected pregnant women. Proceedings of the International AIDS Conference, Barcelona, Spain. July 2002.
10. Minkoff H, Remington JS, Holman SRN et al. Vertical transmission of toxoplasma by human immunodeficiency virus infected women. Am J Obstet Gynecol 1997; 176:555-9.

11. Mandai ON, Lopes FMR, Breganó RM. Prevalence of antibodies IgG and IgM anti-Toxoplasma gondii in pregnant women assisted in the unidades básicas de saúde of Londrina - Paraná, in the period of 2003 and 2004. RBAC 2007; 39(4):247-9.

12. Areal KR, Miranda AE. Seroprevalence of toxoplasmosis in pregnant women attending primary health care in Vitória, ES. NewsLab 2008; 87:122-9.

13. Porto AMF, Amorim MMR, Coelho ICN, Santos LC. Serologic profile of toxoplasmosis in pregnant women attended at a teaching-hospital in Recife. Rev Ass Med Bras 2008; 54(3):242-8.

14. Heukelbach J, Meyer-Cirkel V, Moura RCS et al. Waterborne toxoplasmosis, Northeastern Brazil. Emerg Infect Dis 2007; 13(2):287-9.

15. Galisteu KF, Mattos CB, Lelis AGL et al. Prevalence and risk factors associated with toxoplasmosis in pregnant women and their children in the Northwest of São Paulo State, Brazil. Rev Panam Infectol 2007; 9(4):24-9.

16. Cruz MLS, Cardoso CAC, Saavedra MC, Santos ED, Melino T. Congenital toxoplasmosis infection in an infant born to an HIV-1-infected mother. Braz J Infect Dis 2007; 11(6):610-1.

17. Fauci AS. The human immunodeficiency virus: infectivity and mechanisms of pathogenesis. Science 1988; 617:617-22.

18. Lebech M, Joynson DH, Seitz HM et al. Classification system and case definitions of Toxoplasma gondii infection in immunocompetent pregnant women and their congenitally infected offspring. Eur J Clin Microbiol Infect Dis 1996; 15:799-805.

19. Montoya JG. Laboratory diagnosis of Toxoplasma gondii infection and toxoplasmosis. J Infect Dis 2002; 185(Suppl 1):S73S82.

20. Mozzato L, Procioanoy RS. Incidence of congenital toxoplasmosis in southern Brazil: a prospective study. Rev Inst Med trop S. Paulo 2003; 45(3):147-52. 